\title{
Entre le discours et la réalité : quelques mesures de décentralisation au Québec
}

\author{
Jean-Philippe Meloche et Christopher R. Bryant \\ Université de Montréal
}

\section{La place des gouvernements locaux}

À partir de l'établissement des municipalités en 1840, la structure gouvernementale du Québec se décentralise considérablement. L'immensité du territoire, la faiblesse des communications et le peu d'intervention de l’État central dans l'économie font en sorte que les gouvernements locaux $\mathrm{y}$ assument une part importante des services à la

La décentralisation revient
perpétuellement dans le discours
politique, surtout en temps
de campagnes électorales.

population. Au lendemain de la confédération de 1867, les gouvernements locaux représentent même le palier de gouvernement le plus important en termes de dépenses publiques au Québec.

Dans la structure gouvernementale à trois paliers, établie par l'Acte de l'Amérique du Nord Britannique (1867), les gouvernements locaux n'obtiennent aucun statut constitutionnel propre. Ils relèvent désormais des compétences provinciales. Les deux pôles centraux dans les finances publiques québécoises se consolident alors autour de la capitale provinciale de Québec et de la capitale fédérale d'Ottawa-Gatineau.

Dans la première moitié du $20^{\mathrm{e}}$ siècle, le gouvernement fédéral est très sollicité. Les deux grandes guerres et la grande crise économique des années 1930 le force à jouer un rôle plus important dans les finances publiques. On assiste alors à une importante centralisation des finances publiques vers le palier fédéral. À partir de 1960, parallèlement et en concurrence avec le développement fédéral, il s'opère une modernisation importante au sein de l'État québécois (la Révolution tranquille). Cette modernisation prend la forme d'un rétablissement des forces au profit du palier provincial. D'un point de vue local, il s'agit encore là d'une centralisation des finances publiques.
Vers la fin des années 1970, l’idée de la décentralisation s'installe dans le discours politique au Québec. Le document le plus important qui en témoigne est sans doute le projet de Livre blanc du gouvernement du Québec sur la décentralisation (1978). Même s’il n’a jamais été publié officiellement, ce document lance toutefois le débat sur la question et influence la mise en place de nouvelles structures gouvernementales décentralisées (les MRC). Depuis ce temps, la décentralisation revient perpétuellement dans le discours politique, surtout en temps de campagnes électorales. Elle donne même l'impression d'être un processus en cours. Il n'apparaît toutefois pas évident qu'elle se soit vraiment concrétisée. Comment peuton vérifier si le Québec s'est réellement décentralisé depuis ?

Pour répondre à cette question, il faut d'abord s'entendre sur une définition claire du concept. La décentralisation englobe plusieurs facettes de l'activité gouvernementale. Les multiples définitions qu'on en fait ne sont pas toujours cohérentes les unes avec les autres. À partir d'une définition précise, il est tout de même possible de proposer quelques mesures et de les appliquer au cas du Québec.

\section{Trois formes de décentralisation}

Dans sa définition la plus simple, la décentralisation fait référence à un transfert d'attributions d'un centre vers une périphérie. Elle se concrétise généralement à travers trois différents processus : la déconcentration, la délégation et la dévolution.

La déconcentration est une forme de décentralisation strictement géographique ou administrative. Elle re- 
présente un transfert vers le bas ou vers la périphérie des activités d'un même palier de gouvernement (le palier central). Il s'agit souvent de l'utilisation d'agences ou de bureaux régionaux opérant sous l'autorité du gouvernement central. On l'appelle aussi régionalisation. Les bureaux régionaux de Développement économique Canada (fédéral) ou d’EmploiQuébec (provincial) répartis sur le territoire du Québec représentent des formes de déconcentration.

La délégation et la dévolution réfèrent davantage aux relations intergouvernementales. Elles impliquent un transfert de responsabilités vers des institutions « indépendantes » du gouvernement central. Puisque ces transferts sont principalement d'ordre financier, on parle aussi de décentralisation financière ou fiscale. Dans le cas de la délégation, les responsabilités transférées sont partielles. Elles impliquent souvent un pouvoir de dépenser, sans toutefois garantir de revenus autonomes. Les institutions périphériques restent donc principalement redevables à l'autorité centrale. Les paiements de transferts des gouvernements centraux vers les gouvernements locaux ou les organismes communautaires au Québec représentent divers exemples de délégation.

\section{La décentralisation est impossible à cerner à l'aide d'une seule mesure.}

La dévolution est sans doute la forme la plus complète de décentralisation. Elle implique un transfert d'autorité vers des paliers inférieurs de gouvernement. Les institutions décentralisées acquièrent donc le droit de gouverner leurs propres affaires, incluant le pouvoir de lever des impôts et de voter des budgets sans l'accord de l'autorité centrale. Un transfert d'un point d'impôt ou d'une responsabilité dont le financement peut être assumé de façon autonome par les gouvernements locaux représente un exemple de dévolution.

\section{Mesurer le processus}

La décentralisation est impossible à cerner à l'aide d'une seule mesure. Il existe plutôt une foule d'indicateurs pertinents qui permettent d'en estimer l'ampleur, selon l'une ou l'autre de ses dimensions. Parmi ces indicateurs, certains font appel au degré d'autonomie législative des gouvernements, d'autres réfè- rent à la distribution de ses responsabilités. Les données économiques sur l'emploi et les finances publiques sont parmi les indicateurs les plus accessibles et les plus utilisés. Ce sont d'ailleurs ces indicateurs qui servent ici de cadre d'analyse. Ils ne couvrent évidemment pas tous les aspects de la décentralisation, mais ils en donnent tout de même une représentation générale adéquate.

La manière la plus simple de procéder pour mesurer la décentralisation est d'observer l'évolution des indicateurs sur une période de temps déterminée. Si le poids relatif des gouvernements locaux augmente par rapport aux gouvernements centraux, ou si la taille relative de la périphérie gagne sur les centres, on peut dire qu’il y a présence de décentralisation.

Il faut toutefois faire attention, car ce type de mesure s'exprime en termes relatifs. Pour mesurer la décentralisation de façon absolue, il faudrait s'assurer que la diminution de responsabilités du centre soit proportionnellement compensée par une augmentation des « mêmes » responsabilités dans la périphérie. Cela demande un examen très minutieux des dépenses et des emplois qui ont été transférés dans tous les champs de compétences des gouvernements. Il s’agit d'un exercice très exigeant, mais qui n'est pas d'une nécessité absolue.

Si l'on cherche à mesurer l'effet global de la décentralisation, les mesures relatives sont amplement suffisantes. Il faut savoir, par contre, qu'elles ne sont pas seulement sensibles aux transferts de responsabilités. L'augmentation ou la diminution des activités d'un palier de gouvernement, toute chose étant égale par ailleurs, aura aussi un impact sur ce type de mesures.

Les indicateurs basés sur les finances publiques permettent de bien cerner les deux processus de décentralisation que sont la dévolution et la délégation. Les recettes autonomes des administrations locales, par exemple, représentent un très bon indicateur de dévolution. Pour ce qui est des données sur les dépenses, elles tiennent non seulement compte de la dévolution, mais également d’une part importante de délégation.

Quant aux indicateurs basés sur l'emploi dans l'administration publique, ils sont plus englobants. Ils tiennent compte de l'ensemble des processus de décentralisation, incluant aussi la déconcentration. 


\section{Les indicateurs de décentralisation au Québec}

\section{L'emploi dans l'administration publique}

Il est possible d'estimer la décentralisation au Québec à l'aide des données du recensement sur la population active du secteur de l'administration publique. Ces données permettent d'observer l'évolution de la concentration géographique de la fonction publique au Québec depuis les années 1970. Elles donnent surtout une idée de la répartition des emplois des gouverne- ments fédéral et provincial sur l'ensemble du territoire (la déconcentration). Comme le montre le tableau 1, la part des régions centrales (Outaouais et Québec) dans l'emploi du secteur de l'administration publique a légèrement augmenté entre 1971 et 2001, passant de $31,5 \%$ à $35 \%$. Ces données montrent clairement que la fonction publique s'est concentrée davantage dans les régions centrales au cours de cette période, au détriment de la périphérie. Elles laissent donc présager qu'il y aurait eu centralisation plutôt que décentralisation gouvernementale au Québec depuis les années 1970.

Tableau 1 - Parts des régions administratives de l'Outaouais et de Québec dans la population active du secteur de l’administration publique au Québec, 1971 à 2001 (en \%)

\begin{tabular}{cccc} 
& Outaouais & Québec & Total \\
\hline $\mathbf{1 9 7 1}$ & 9,2 & 22,3 & 31,5 \\
$\mathbf{1 9 8 1}$ & 11,1 & 21,9 & 33,1 \\
$\mathbf{1 9 9 1}$ & 12,7 & 20,2 & 32,9 \\
$\mathbf{2 0 0 1}$ & 14,9 & 20,1 & 35,0
\end{tabular}

Source : Statistique Canada, Données de recensement. Compilation du Bureau de la Statistique du Québec pour 1971 et 1981 et de l'Institut de la Statistique du Québec pour 1991 et 2001.

Il est vrai que la part relative de la région de Québec (Capitale-Nationale) dans la population active du secteur de l'administration publique a diminué au cours de la période, passant de 22,3 \% à 20,1\%. Cette diminution est toutefois largement compensée par le gain relatif de l'Outaouais qui passe de 9,2 \% à 14,9\% sur la même période. Le nombre de personnes actives dans le secteur de l'administration publique a crû de $163 \%$ dans l'Outaouais entre 1971 et 2001, alors qu'il ne s'est accrû que de 46 \% à Québec et de 54 \% dans le reste du Québec.

\footnotetext{
L'administration publique sur le territoire québécois s'est relativement centralisée entre 1971 et 2001 , essentiellement vers l'Outaouais, malgré une déconcentration de la fonction publique québécoise depuis la capitale provinciale.
}

Ce gain relatif de la région administrative de l'Outaouais s'explique toutefois davantage par un transfert d'emplois depuis la région ontarienne d'Ottawa que par une centralisation de l'emploi fédéral au Québec. L'augmentation de la part relative du Québec dans l'emploi public fédéral a donc une influence considérable sur la mesure de décentralisation. Dans tous les cas, le résultat est toutefois le même, une plus grande part de la population active du secteur de l'administration publique au Québec se concentre dans la région administrative de l'Outaouais. On peut alors conclure que l'administration publique sur le territoire québécois s'est relativement centralisée entre 1971 et 2001, essentiellement vers l'Outaouais, malgré une déconcentration de la fonction publique québécoise depuis la capitale provinciale.

\section{Les finances publiques locales}

Les données sur les finances publiques offrent également une bonne perspective sur la décentralisation. Le 
tableau 2 présente l'évolution des parts relatives de chacun des paliers de gouvernement dans l'ensemble des finances publiques au Québec entre 1973 et 2003. On y voit très clairement, autant en termes de recettes qu'en termes de dépenses, que la part relative des gouvernements locaux y diminue à travers le temps.
Cette diminution n'est pas constante, mais elle semble bien établie. On ne peut donc pas conclure qu'il y ait présence de décentralisation dans les finances publiques au Québec depuis les années 1970. Bien au contraire, la tendance semble même plutôt à la centralisation.

\section{Tableau 2 - Évolution des parts des paliers gouvernementaux dans les finances publiques du Québec 1973-2003 ${ }^{1}$}

\begin{tabular}{|cccc|ccc}
\hline & \multicolumn{2}{c}{$\begin{array}{c}\text { Part des recettes publiques } \\
\text { avant transferts (\%) }\end{array}$} & \multicolumn{3}{c}{$\begin{array}{c}\text { Part des dépenses publiques } \\
\text { après transferts (\%) }\end{array}$} \\
\hline & Local & Provincial & Fédéral & Local & Provincial & Fédéral \\
\hline $\mathbf{1 9 7 3}$ & 11,3 & 41,0 & 47,6 & 24,2 & 39,8 & 60,0 \\
$\mathbf{1 9 8 3}$ & 10,6 & 49,0 & 40,3 & 18,4 & 44,5 & 37,0 \\
$\mathbf{1 9 9 3}$ & 11,4 & 47,7 & 41,0 & 16,8 & 46,4 & 36,8 \\
$\mathbf{2 0 0 3}$ & 8,6 & 50,7 & 40,7 & 14,2 & 52,5 & 33,3 \\
& & & & & &
\end{tabular}

Source : Institut de la statistique du Québec, Comptes économiques annuels, Édition 2004. Statistique Canada, Recettes et dépenses des administrations fédéral, provinciales et locales.

C'est le gouvernement provincial qui se taille la part du lion dans les finances publiques au Québec. Il est d'ailleurs le seul à y avoir augmenté sa part depuis les années 1970. L’évolution de ses portefeuilles, notamment celui de la santé, lui procure une croissance beaucoup plus prononcée que celle des autres paliers de gouvernement. On comprend donc que le statu quo en matière de compétences a aussi un impact considérable sur la décentralisation gouvernementale au Québec. Sans qu'aucun transfert de responsabilités ne soit effectué, les finances gouvernementales tendent à se concentrer davantage entre les mains du gouvernement dont les besoins augmentent le plus rapidement. $\mathrm{Au}$ Québec, ce phénomène alimente la centralisation des finances publiques vers le palier provincial.

Bien que le nombre d'indicateurs présentés ici soit limité, il est tout de même possible de tirer des conclusions sommaires sur la décentralisation au Québec. Tous les indicateurs pointent d'ailleurs dans la même direction : le gouvernement ne semble pas plus décentralisé aujourd’hui qu'au début des années 1970.

\section{Le Québec : État décentralisé ?}

Il est possible que le Québec ne se soit jamais réellement décentralisé sous prétexte que son niveau de décentralisation est amplement suffisant. Sachant qu'il n'existe aucune règle absolue qui permette de fixer un niveau de décentralisation suffisant, il est difficile de vérifier cette affirmation. Règle générale, on dit d'un État qu'il est plus ou moins décentralisé en le comparant à d'autres États qui lui sont similaires. Où se situe donc le Québec dans une telle comparaison?

\section{C'est le gouvernement provincial qui se taille la part du lion dans les finances publiques au Québec. Il est d'ailleurs le seul à y avoir augmenté sa part depuis les années 1970.}

À l'aide de données sur les finances publiques, il est possible de comparer la structure gouvernementale du Québec à celle d'autres États. Les données sur les finances publiques n'englobent pas toutes les dimensions de la décentralisation. Elles ne tiennent pas compte notamment de la déconcentration. Elles représentent toutefois la meilleure base de comparaison entre les États. Elles donnent aussi une idée relativement juste des processus de dévolution et de délégation. 
Le Québec est une province au sein du Canada. Les États qui lui sont les plus similaires en termes de fonctionnement sont donc sans doute les autres provinces canadiennes. Le tableau 3 offre une comparaison des parts relatives des paliers local et provincial dans les finances publiques des provinces pour l'ensemble du Canada, excluant les finances du gouvernement fédéral. On y constate que la part des gouvernements locaux est relativement plus faible au Québec que dans la majorité des autres provinces canadiennes. Seuls les gouvernements locaux des pro- vinces maritimes ont des parts plus faibles dans leurs finances publiques que le Québec. Le Québec se retrouve même sous la moyenne fédérale. L’Ontario est la province où les gouvernements locaux occupent la plus grande part des finances publiques et son poids démographique affecte considérablement la moyenne canadienne. Cette comparaison interprovinciale permet tout de même de conclure que le Québec n'est pas une province nécessairement plus décentralisée que les autres.

Tableau 3 - Part des gouvernements locaux dans les finances publiques, excluant le gouvernement fédéral, selon les provinces du Canada, 2003

\begin{tabular}{lcccc} 
Provinces & \multicolumn{2}{c}{$\begin{array}{c}\text { Part des recettes publiques } \\
\text { avant transferts (\%) }\end{array}$} & \multicolumn{2}{c}{$\begin{array}{c}\text { Part des dépenses publiques } \\
\text { après transferts (\%) }\end{array}$} \\
\hline Québec & Local & Provincial & Local & Provincial \\
\hline Terre-Neuve & $\mathbf{1 3 , 4}$ & $\mathbf{8 6 , 6}$ & $\mathbf{2 3 , 8}$ & $\mathbf{7 6 , 2}$ \\
Île du Prince Édouard & 5,2 & 94,8 & 18,7 & 81,3 \\
Nouvelle-Écosse & 4,8 & 95,2 & 16,9 & 83,1 \\
Nouveau-Brunswick & 10,6 & 89,4 & 22,1 & 77,9 \\
Ontario & 7,5 & 92,5 & 10,1 & 89,9 \\
Manitoba & 23,0 & 77,0 & 35,5 & 64,5 \\
Saskatchewan & 14,2 & 85,8 & 24,1 & 75,9 \\
Alberta & 17,1 & 82,9 & 24,2 & 75,8 \\
Colombie-Britannique & 17,7 & 82,3 & 30,5 & 69,5 \\
Canada & 14,8 & 85,2 & 25,0 & 75,0 \\
& $\mathbf{1 7 , 2}$ & $\mathbf{8 2 , 8}$ & $\mathbf{2 8 , 3}$ & $\mathbf{7 1 , 7}$
\end{tabular}

Source : Statistique Canada, Recettes et dépenses des administrations provinciales et locales.

Le fait que le Québec ne soit pas plus décentralisé que les autres provinces canadiennes ne signifie pas nécessairement qu'il ne soit pas un État décentralisé. Le Canada peut être un pays où les gouvernements locaux occupent une part relativement importante des finances publiques. Le Québec serait-il donc un État décentralisé dans un État encore plus décentralisé que lui, le Canada? Pour répondre à cette question, on peut tenter de positionner le Québec dans une perspective internationale. Il faut toutefois faire attention au genre de comparaison qu'on fait à cette échelle. Le Québec n'est pas un état souverain mais une province dans un état fédéré. Il n’existe pas deux niveaux de gouvernement au Québec, mais trois. Cette caractéristique est commune aux États fédérés. On la retrouve au Canada, mais aussi en Australie, en Allemagne, en
Autriche, en Belgique, au Mexique, en Suisse et aux États-Unis (pour ne nommer que ceux qui sont membres de l'OCDE). Les États unitaires, de leur côté, n'ont que deux paliers de gouvernement : local et central. En l'absence de structures intermédiaires, il serait normal que les gouvernements unitaires renvoient plus de responsabilités vers leurs gouvernements locaux, c'est pourquoi il est difficile de les comparer avec le Québec.

Le tableau 4 présente l'importance relative des différents paliers de gouvernement dans les finances publiques pour le Québec, le Canada et sept autres pays fédérés membres de l'OCDE. Il faut y voir une comparaison entre le Québec, qui est une province, et la moyenne des provinces canadiennes ou la moyenne 
des États sous-nationaux des autres pays fédérés. On y constate que la part des gouvernements locaux dans les recettes publiques est relativement faible au Québec. La part du gouvernement fédéral y est également relativement faible, ce qui indique une présence nettement plus grande du palier provincial (palier intermédiaire) au Québec que pour la moyenne des États sous-nationaux des autres pays fédérés. Ainsi, la concentration gouvernementale au Québec serait relativement plus orientée vers le gouvernement provincial que vers le gouvernement fédéral.
On remarque également que le retard dans les revenus des gouvernements locaux au Québec est relativement compensé par les paiements de transferts. La part des gouvernements locaux dans les dépenses publiques au Québec est semblable à celle de la plupart des États sous-nationaux des autres pays. Le Québec serait donc aussi décentralisé que ces derniers en termes de dépenses. Le retard, dans ce cas, ne serait pas tant au niveau de la décentralisation globale qu'à celui de la dévolution (les revenus autonomes servant d'indicateurs de dévolution).

Tableau 4 - Comparaison internationale des parts des paliers gouvernementaux dans les finances publiques, pays fédérés et Québec, 2001

\begin{tabular}{lccc|ccc}
\multicolumn{1}{c}{ Pays } & \multicolumn{2}{c|}{$\begin{array}{c}\text { Part des recettes publiques } \\
\text { avant transferts (\%) }\end{array}$} & \multicolumn{2}{c}{$\begin{array}{c}\text { Part des dépenses publiques } \\
\text { après transferts (\%) }\end{array}$} \\
\hline & Local & Provincial & Fédéral & Local & Provincial & Fédéral \\
\hline Québec & 8,6 & 49,6 & 41,8 & 14,9 & 51,0 & 34,1 \\
Canada & 11,4 & 40,9 & 47,6 & 16,2 & 44,3 & 39,5 \\
Australie & 5,5 & 20,9 & 73,6 & 6,3 & 38,4 & 55,3 \\
Autriche & 15,1 & 9,3 & 75,6 & 15,5 & 16,3 & 68,2 \\
Allemagne & 11,1 & 22,9 & 66,0 & 15,0 & 22,5 & 62,5 \\
Belgique & 6,9 & 5,5 & 87,6 & 12,5 & 21,9 & 65,5 \\
Mexique* & 6,1 & 17,9 & 75,9 & 5,2 & 15,1 & 79,7 \\
Suisse & 24,2 & 26,5 & 49,3 & 23,9 & 32,0 & 44,1 \\
États-Unis & 16,6 & 23,4 & 60,0 & 25,7 & 22,8 & 51,6
\end{tabular}

Sources : Institut de la statistique du Québec (pour le Québec). Fond Monétaire International, Government Finance Statistics Yearbook, 2004 (pour les autres pays).

* Données de l’année 2000.

Il faut cependant être prudents avec les données internationales sur les finances publiques (qui proviennent du Government Finance Statistics Yearbook, FMI). Ces données ne permettent pas de déterminer si les recettes ou les dépenses des gouvernements sousnationaux sont gérées de façon autonome, pas plus qu'elles ne tiennent compte des caractéristiques propres à chaque système d'administration publique. La façon d’attribuer une dépense publique au niveau local peut aussi être sujet à débat.

Ces données restent toutefois les seules disponibles à ce jour qui permettent une quelconque comparaison internationale des finances publiques. C'est pourquoi elles sont utilisées ici.

\section{La concentration gouvernementale au Québec serait relativement plus orientée vers le gouvernement provincial que vers le gouvernement fédéral.}

\section{Conclusion}

Bien que la décentralisation fasse partie du discours politique au Québec depuis près de trois décennies, rien ne semble avoir bougé dans les structures gouvernementales. La répartition de l'emploi et des finances publiques y semble même plus centralisée que jamais. Au niveau fédéral, la restructuration des entre- 
prises publiques et le transfert des emplois d'Ottawa vers Gatineau auront eu pour effet de diminuer les ressources fédérales au Québec, tout en les centralisant davantage sur l'Outaouais. Quant au gouvernement provincial, ses champs de compétences, principalement la santé, justifient sa croissance soutenue dans les finances publiques. Ses efforts de déconcentration amoindrissent quelque peu l'effet de la centralisation. Le statu quo au plan des compétences y maintient toutefois une pression constante. On peut donc s’attendre, si le gouvernement du Québec ne revoit pas sérieusement le partage de ses responsabilités avec les gouvernements locaux, à ce que les finances publiques tendent à se centraliser encore davantage dans l'avenir. Ce constat est d'autant plus sérieux que le Québec apparaît déjà comme un État relativement centralisé.

\section{Note}

1 Les recettes publiques avant transferts représentent l'ensemble des revenus de source autonome des gouvernements. Quant aux dépenses après les transferts, elles représentent les dépenses budgétaires totales à l'exclusion des paiements de transferts. La différence entre les deux mesures équivaut aux paiements de transferts. 


\section{Publicité}

Maîtrise en gestion de projet 